\title{
Incentive effects of funding contracts: an experiment
}

\section{J. Philipp Reiß • Irenaeus Wolff}

\begin{abstract}
We examine the incentive effects of funding contracts on entrepreneurial effort and on allocative efficiency. We experiment with funding contracts that differ in the structure of investor repayment and, thus, in their incentives for the provision of entrepreneurial effort. Theoretically the replacement of a standard debt contract by a repayment-equivalent non-monotonic contract reduces effort distortions and increases efficiency. Likewise, distortions can be mitigated by replacing outside equity by a repayment-equivalent standard-debt contract. We test both hypotheses in the laboratory. Our results reveal that the incentive effects of funding contracts must be experienced before they are reflected in observed behavior. With sufficient experience, observed behavior is either consistent with or close to theoretical predictions and supports both hypotheses. If we allow for entrepreneur-sided manipulations of project outcomes, we find that non-monotonic contracts lose much of their appeal.
\end{abstract}

Keywords Hidden information · Funding contracts · Incentives · Experiment · Standard debt contract $\cdot$ Non-monotonic contract $\cdot$ State manipulation

Financial support from Maastricht University through METEOR is gratefully acknowledged. We thank Paul Smeets and audiences in Alicante (IMEBE2008), Caltech (ESA2008), Gothenburg (ESEM2013), Heidelberg, Luxembourg (GfEW2010), and Lyon (ESA2008) for helpful comments. The paper greatly benefited from helpful suggestions and comments of Jordi Brandts and two anonymous reviewers.

\section{J.P. Reiß $(\varangle)$}

Institute of Economics (ECON), Karlsruhe Institute of Technology (KIT), Schlossbezirk 14,

76131 Karlsruhe, Germany

e-mail: philipp.reiss@kit.edu

I. Wolff

TWI/University of Konstanz, Hauptstr. 90, 8280 Kreuzlingen, Switzerland

e-mail: wolff@twi-kreuzlingen.ch 
JEL Classification C91 $\cdot$ D $82 \cdot$ G21

\section{Introduction}

There are many real-life instances where individuals have an opportunity to engage in a "project" that yields risky returns. Examples range from starting a business to pursuing a university degree. Typically project execution requires fixed setup costs that exceed the available funds of the project's owner-manager (henceforth entrepreneur) and are thus financed by outside investors, e.g. lenders, shareholders, or the government (in case of government subsidy programs).

The specified terms of repayment to the investor form an integral element of outside financing and can differ considerably among differing financing arrangements. For example, (1) government agencies subsidizing unemployed workers to start businesses may require no repayment at all; (2) an entrepreneur may take out a loan requiring repayment of either a constant amount or all available assets in case of bankruptcy; (3) student loan programs may request that students repay less than the loan amount if they are more successful in their studies than their fellow students; (4) all potential returns to entrepreneurship may be divided into specified shares between the entrepreneur and the investor.

As an entrepreneur can improve the prospects of high returns by exerting more effort, the division, fixed in the funding contract, of as yet uncertain returns between an entrepreneur and an investor can potentially affect the entrepreneur's effort choice. This raises the question of how incentives inherent in funding contracts shape entrepreneurial outcomes. This question matters, as misallocations of external funding or suboptimal incentivization of entrepreneurs can lead to static and dynamic welfare losses. The latter can result because static inefficiencies may inhibit the growth of per capita output, which relies on technical advance, to which entrepreneurial innovative contributions are key. ${ }^{1}$

The seminal paper by Innes (1990) provides a thorough theoretical analysis of how entrepreneurial outcomes are shaped in a setting of external finance with hidden effort and limited liability. Recent research on behavioral corporate finance, however, demonstrates that theoretical predictions under the self-interest-hypothesis paired with full rationality can systematically deviate from empirical outcomes. ${ }^{2}$ The purpose of this paper is to investigate experimentally how funding contracts influence entrepreneurial behavior and to inquire into the implications of funding contracts on allocative efficiency in an attempt to improve our understanding of the scope and extent of entrepreneur-sided behavioral effects in our setting of external finance.

\footnotetext{
${ }^{1}$ See, e.g., Romer (1990) and Aghion and Howitt (1992). For textbook treatments of growth economics, see, e.g., Aghion and Howitt (1998) and Barro and Sala-i-Martin (2003).

${ }^{2}$ For example, Malmendier and Tate (2005) have shown that CEO overconfidence can lead to corporate investment distortions among Forbes 500 CEO's. More generally, Baker and Wurgler (2012) and Baker et al. (2007) survey literature in behavioral corporate finance that distinguishes between investor-sided and manager-sided behavioral effects. Although these empirical studies investigate settings that are fundamentally different from our entrepreneur-based external finance setting, they show that theoretical predictions, given the assumptions of self-interest and full rationality, require careful empirical and/or experimental evaluation, also in corporate finance settings.
} 
Innes (1990) theoretically shows that standard debt contracts induce inefficiently low effort, leading to substantial efficiency losses. In contrast, non-monotonic contracts can overcome this problem, under a wide range of parameter values (non-monotonic-contracts hypothesis). If designed accordingly, non-monotonic contracts can induce efficient effort choices. Furthermore, Innes (1990) demonstrates that standard-debt contracts, though inefficient, are more efficient than any other repayment-equivalent monotonic repayment contract in the class of monotonic contracts (monotonic-contracts-hypothesis). In our experiment, we set out to test both hypotheses. To test the non-monotonic-contracts hypothesis, we compare behavior under a standard debt contract to behavior under a non-monotonic contract yielding the same expected repayment to the investor. To test the monotonic-contracts hypothesis, we compare a standard debt contract to a repayment-equivalent outside equity contract. To obtain a more complete picture of the incentive effects of funding contracts, we also study behavioral responses under no-repayment contracts as a benchmark. ${ }^{3}$ As noted by Innes (1990, p. 46), practical disadvantages arise with nonmonotonic contracts if contracting parties have opportunities to manipulate states, e.g., through investor-sided sabotage of the entrepreneurial project or entrepreneursided outside borrowing. We experimentally test a non-monotonic contract also in a broader environment where entrepreneurs can misreport return states as a reduced form of outside borrowing.

We find that the incentive effects of funding contracts are too subtle to be grasped by introspection alone. In the early rounds of the experiment, we find no differences at all in entrepreneurial behavior across contract conditions. In addition, entrepreneurial behavior is quite similar in a one-shot treatment in which the stakes are raised by a factor of fifteen, showing that experience with incentives is indispensable. This is of particular interest in the studied setting, as many real-life entrepreneurs are similarly inexperienced when relying on external finance for the first, and possibly only, time. Nevertheless the theoretical predictions strongly attract behavior over the course of the experiment. With accumulating experience, behavior moves closer to the theoretical point predictions, and the comparative statics predictions apply across funding contracts. At the end of the experiment, behavior is either consistent with or close to the theoretical point predictions.

More specifically, we find support for the non-monotonic contracts hypothesis, if experience with contract incentives is sufficient. Allocative efficiency is much greater under a non-monotonic contract than under a repayment-equivalent standard debt contract, as incentives under the former induce first-best behavior. Because, under a non-monotonic contract, repayment to the investor is less in higher return states, reliable state verification and enforcement is predicted to be essential to successful implementation of a non-monotonic contract. Allowing for the opportunity to misrepresent states in the experiment shows that entrepreneurs increasingly misrepresent states to their advantage. Although the observed magnitude of misrepresentation is less than the potential magnitude of misrepresentation, it is sufficient

\footnotetext{
${ }^{3}$ For simplicity we refer to cases where the entrepreneur retains the full return on a project as no-repayment contracts, even if no explicit repayment contract is written. Examples would include cases where the entrepreneurial project is fully subsidized or where entrepreneurs do not rely on external finance but selffinance instead.
} 
to erode the investor's participation constraint. Thus, our experiment confirms the importance of state verification. We also find that, if experience with contract incentives is sufficient, behavior in the experiment under an outside equity contract deviates more from first-best behavior than behavior under a repayment-equivalent standard-debt contract. Thus, we find support for the monotonic-contracts hypothesis.

This paper contributes to the growing experimental literature on credit markets, as, to the best of our knowledge, this study is the first to examine the incentive effects of funding contracts and the non-monotonic-contracts and monotonic-contracts hypotheses. An experimental study related to ours is Serra-Garcia (2010), that explores the effects of collateral. It reports a positive relationship between collateral and entrepreneurial effort, one that, in contrast to standard theory, emerges only if repayment to the investor is sufficiently low. Other experimental studies of credit markets include Brown and Zehnder (2007, 2010), who investigate the effect of information dissemination of loan defaults on repayment behavior, and Fehr and Zehnder (2006), who study the role of reputation in credit markets.

Our findings also complement the literature on moral hazard. ${ }^{4}$ In this literature, it is natural to model the principal-agent relationship in such a manner that the residual claimant owns the project (principal) and — to execute the project—another party (agent) provides an unobserved input (effort). Our setting, by contrast, allows us to explore the diametral case in which the residual claimant, who owns the project, is also the contract party who provides the unobservable input (effort). This assumption is natural in our setting, as the entrepreneur owns the project and executes it. A second party (the investor) is also required here, as execution of the project requires provision of an indispensable input (external funding) that the entrepreneur lacks.

The paper is organized as follows. Section 2 provides the theoretical foundation of our experimental research. Section 3 summarizes the experimental design. Section 4 reports our experimental results. Section 5 concludes.

\section{Theoretical considerations}

In this section, we begin by outlining a simple model of entrepreneurial external financing that serves as the foundation for our experimental investigation. We then introduce the basic structures of repayment contracts and their incentive effects. We review contract structures that are either frequently observed in real-life or are optimal in our setting with costless state verification. In addition, in introducing repay-

\footnotetext{
${ }^{4}$ For example, DeJong et al. (1985) demonstrate the relevance of moral hazard with flat wage employment contracts. Fehr et al. (1993) and Irlenbusch and Sliwka (2005) show that agents' effort levels increase in the generosity of flat wages. Fehr et al. (2007), on the other hand, report that bonus contracts outperform flat wage contracts, while Brandt and Charness (2004) investigate the impact of competitive imbalances and minimum wages. Contract design has also been shown in the field to affect behavior. For example, Lazear (2000) finds that replacing flat rate hourly pay by piece rates, for windshield installers, increases productivity, while Shearer (2004) reports a similar effect for workers in tree-planting.
} 
ment contracts, we also note two fundamental theoretical results, the non-monotoniccontracts hypothesis and the monotonic-contracts hypothesis.

\subsection{A simple model of funding}

The outlined model is a discrete variant of Innes (1990). Consider an entrepreneurial project with a random return $Z$. The underlying probability function is such that greater entrepreneurial effort increases the likelihood of outcomes with high returns. There are $n$ return states. The project return in state $i$ is denoted by $z_{i} \geq 0$. Return states are numbered in ascending order, i.e. $z_{i}<z_{j}$ if $i<j$. The probability of state $i$ depends on entrepreneurial effort $x \in[0, \bar{x}]$ and is given by $p_{i}(x) \geq 0$, where $p_{i}(x)$ is twice-differentiable. For a proper probability distribution assume $\sum_{i} p_{i}(x)=1$ and $\sum_{i} p_{i}^{\prime}(x)=0$. To capture the idea that greater effort increases the probability of a higher return state, suppose that the monotone likelihood ratio property (MLRP) is satisfied, i.e., for all $z_{i}<z_{j}$, we have $p_{i}^{\prime}(x) / p_{i}(x)<p_{j}^{\prime}(x) / p_{j}(x)$, implying $\partial E[Z \mid x] / \partial x>0$, cf. Milgrom (1981). To ensure an interior solution, we assume that the marginal benefit of effort does not grow to infinity, i.e., $\lim _{x \rightarrow \bar{x}} \partial E[Z \mid x] / \partial x$ is finite.

The project has start-up cost $\Gamma>z_{1} .{ }^{5}$ The entrepreneur is endowed with wealth $W$. The amount of external finance required to start the project is $D \equiv \Gamma-W$. As we inquire into the effects of external financing schemes on entrepreneurial activity, we assume that $D>0$. For simplicity, let $W=0$. We assume the entrepreneur is subject to limited liability, such that the realized project return constrains repayment in low return states. A feasible repayment contract $\vec{t}$ is characterized by $\vec{t}=\left(t_{1}, t_{2}, \ldots, t_{n}\right)$, such that $t_{i} \leq z_{i}$ (due to limited liability), where $t_{i}$ denotes the contracted amount of repayment in state $i$.

The preferences of the entrepreneur are additively separable in income $y$ and in the cost of effort, $c(x)$ :

$$
u(x, y)=y-c(x)
$$

where $c(0)=0, c^{\prime}(x)>0, c^{\prime \prime}(x)>0$, and $\lim _{x \rightarrow \bar{x}} c^{\prime}(x)=\infty$. As the entrepreneur's income in state $i$ is the difference between the realized project return and contracted repayment, the entrepreneur's maximization problem for any given contract $\vec{t}$ is given by:

$$
\max _{x} E U(x, \vec{t})=\sum_{i=1}^{n} p_{i}(x)\left(z_{i}-t_{i}\right)-c(x) .
$$

Expected utility is maximized by effort level $\tilde{x}(\vec{t})$. For ease of exposition, let $c(x)$ be sufficiently concave to always guarantee strict concavity of the objective function. The first-order condition of the maximization problem then characterizes a unique

\footnotetext{
${ }^{5}$ If the start-up cost is not larger than the lowest project return $z_{1}$, the financing problem is trivial.
} 
global maximum of entrepreneurial expected utility: ${ }^{6}$

$$
\sum_{i=1}^{n} p_{i}^{\prime}(\tilde{x}) z_{i}=c^{\prime}(\tilde{x})+\sum_{i=1}^{n} p_{i}^{\prime}(\tilde{x}) t_{i}
$$

The LHS of (1) gives the marginal expected project return of additional effort. The first term on the RHS is the marginal cost of effort.

The key to understanding how entrepreneurial incentives are related to funding contracts lies in the second term on the RHS: the marginal expected repayment to the lender, $\operatorname{MR}(x) \equiv \sum p_{i}^{\prime}(x) t_{i}$. If the repayment contract implies that the marginal expected repayment vanishes from (1), the entrepreneur finds it optimal to supply first-best effort $x^{*}$ that prevails in the absence of external financing $\left(t_{i}=0 \forall i\right)$; hence, any flat contract $\left(t_{i}=\tau \forall i\right)$ induces first-best effort. ${ }^{7}$

If, however, the funding contract is designed so that the marginal expected repayment does not sum to zero, the funding contract distorts the entrepreneur's choice of effort level, leading to an inefficient effort provision and a loss of economic surplus. Specifically, the entrepreneur's optimal effort $\tilde{x}$ decreases in the marginal expected repayment, as application of the implicit function theorem to $\tilde{x}=f(\mathrm{MR})$, implicitly defined by (1), shows:

$$
\frac{d \tilde{x}}{d \mathrm{MR}}=\frac{1}{\sum_{i=1}^{n} p_{i}^{\prime \prime}(\tilde{x}) z_{i}-c^{\prime \prime}(\tilde{x})-\mathrm{MR}^{\prime}(\tilde{x})}<0 .
$$

The denominator is the maximization problem's second-order condition, so that a strictly positive marginal expected repayment implies that $\tilde{x}<x^{*}$, resulting in lost economic surplus.

\subsection{Types of repayment contracts}

As the incentive effects of funding contracts are reflected in the marginal expected repayment to the investor, they are influenced by the structures of the funding contracts. We distinguish between four basic repayment contract structures that differ in the way that state-contingent repayments vary with higher project returns: flat contracts, standard debt contracts, non-monotonic contracts (hill-shaped), and outside equity contracts. We single out these structures, as they are either widely employed in real-life or constitute the optimal contract structure in our setting. In the following subsections, we describe these contract structures in more detail, discuss their incentive effects, and review the non-monotonic-contracts hypothesis and the monotoniccontracts hypothesis.

\footnotetext{
${ }^{6}$ Due to the generality of feasible contracts and revenue distributions, it is possible to find contracts that imply a strictly negative marginal entrepreneurial income net of repayment, even with zero effort (e.g., a contract that always requires full repayment, except in the lowest return state, where no repayment is required). It is then impossible to satisfy the first-order condition (1), and a boundary solution emerges in which $\widetilde{x}(\vec{t})=0$.

${ }^{7}$ Recall that $\sum p_{i}^{\prime}(x)=0$; otherwise, the probabilities would sum to more or less than unity with variations in effort.
} 


\subsubsection{Flat contracts and the no-repayment contract}

A flat contract is fully specified by a constant payment $\tau \geq 0$ that the entrepreneur repays to the investor independently of the realized return state; hence, $t_{i}=\tau$. In our setting, feasible flat contracts satisfy $\tau \leq z_{1}$, due to limited liability. As repayment to the investor does not vary with the effort level of the entrepreneur, the marginal repayment under a flat contract is zero, so that, trivially, any flat contract induces first-best effort. Below, we experimentally study the no-repayment contract $\vec{t}$ NoRepay, with $\tau=0$, which is a special case of a flat contract.

\subsubsection{The standard debt contract}

A widely applied funding contract is the standard debt contract, which essentially reduces the repayment structure to a flat repayment claim $\tau$ that is independent of the realized return state. However, due to binding limited liability, the actual repayment to the lender is smaller than $\tau$, whenever the realized project return falls short of the flat repayment claim. Using our contract notation, a standard debt contract $\vec{t}$ SDC is given by

$$
t_{i}^{\mathrm{SDC}}= \begin{cases}z_{i} & \text { if } z_{i}<\tau \\ \tau & \text { otherwise }\end{cases}
$$

Under a standard debt contract, the entrepreneur shares with the lender the benefit of increased expected project return generated by additional effort while bearing the total marginal cost of effort. A key characteristic of this type of contract is that the implied marginal expected repayment is strictly positive, so that the standard debt contract is inherently inefficient. To see this, note that the expected repayment to the lender, under any standard debt contract, is given by $\sum_{i=1}^{m-1} p_{i}(x) z_{i}+\sum_{i=m}^{n} p_{i}(x) \tau$ where $m$ is the smallest payoff state that allows the entrepreneur to fully repay the fixed payment of the standard debt contract. Rewriting the expected repayment and differentiating it with respect to effort yields the marginal expected repayment as

$$
\begin{aligned}
\operatorname{MR}^{\mathrm{SDC}}(x)= & z_{i} \sum_{i=1}^{n} p_{i}^{\prime}+\left(z_{2}-z_{1}\right) \sum_{i=2}^{n} p_{i}^{\prime}+\cdots+\left(z_{m-1}-z_{m-2}\right) \sum_{i=m-1}^{n} p_{i}^{\prime} \\
& +\left(\tau-z_{m-1}\right) \sum_{i=m}^{n} p_{i}^{\prime} .
\end{aligned}
$$

By definition of a proper distribution function, the sum of marginal probabilities equals zero, $\sum_{i=1}^{n} p_{i}^{\prime}=0$, so that the first summation vanishes. All other summations differ from the first one in that the marginal probabilities of low revenue states are not included in these summations. The property that increased effort reduces the probability of low return states and increases that of high return states implies that the lowest payoff states are assigned negative marginal probabilities; thus, when they are omitted, all remaining summations are strictly positive. It follows that the marginal expected repayment under any standard debt contract is always strictly positive, and, thus, the induced entrepreneurial effort choice is suboptimal. 


\subsubsection{Optimal non-monotonic contracts and the non-monotonic-contracts hypothesis}

Although standard debt contracts are inefficient, it is possible to design Paretoimproving contracts that can overcome the inefficiency inherent in standard debt contracts. These contracts are characterized by a non-monotonic repayment structure in the sense that repayment in some higher-return states is lower than repayment in some lower-return states. By decreasing repayment in high-return states, the marginal repayment to the lender - which is strictly positive under standard debt contracts - is reduced, while the expected repayment to the lender is preserved. It follows that the deviation from first-best effort and the implied efficiency loss with non-monotonic contracts are smaller than under standard debt contracts, due to better incentives provided by the former. If designed accordingly, non-monotonic contracts can even lead the entrepreneur to exert first-best effort, thus eliminating any efficiency loss (Proposition 1). A numerical example that illustrates the potential magnitude of welfare gains through non-monotonic contracts, which we experimentally investigate, is provided in Sect. 3.

Proposition 1 (Non-monotonic-contracts hypothesis) There may exist non-monotonic contracts that are superior to standard debt contracts in terms of entrepreneurial profit and allocative efficiency, due to a smaller deviation from first-best effort.

Proof omitted.

\subsubsection{Outside equity contracts and the monotonic-contracts hypothesis}

Outside equity contracts are a special case of monotonic contracts where repayment to the investor is higher if the entrepreneur's revenue realization is higher, $t_{i}<t_{j}$ if $i<j$. For outside equity contracts, the share of investor repayment in revenue is the same, regardless of the return state. We denote the share of investor repayment by $\sigma \in(0,1]$ and refer to it as the equity share. Then any outside equity contract $\vec{t}$ Equi is defined by

$$
t_{i}^{\text {Equi }}=\sigma z_{i} \quad(i=1, \ldots, n)
$$

To pin down the contract incentives of outside equity contracts, we derive the marginal expected repayment of outside equity contracts. By MLRP there is a state $q \in\{1, \ldots, n\}$, such that $p_{i}^{\prime}<0<p_{j}^{\prime}$ and $p_{q}^{\prime} \geq 0$ for all $i<q<j$, i.e., all states with project returns larger than $z_{q}$ become more likely with increased effort, while all states with project returns smaller than $z_{q}$ become less likely. Differentiating the expected repayment under outside equity, $R^{\text {Equi }}=\sigma \sum_{k=1}^{n} p_{k}(x) z_{k}$, and grouping terms by the sign of marginal probabilities leads to the marginal expected repayment under any outside equity contract as follows:

$$
\operatorname{MR}^{\text {Equi }}(x)=\sigma \cdot\left[\sum_{i=1}^{q-1} p_{i}^{\prime} z_{i}+\sum_{j=q}^{n} p_{j}^{\prime} z_{j}\right]>0
$$


where the first summation sums over strictly negative terms and the second summation sums over positive terms. As all marginal probabilities sum up to zero and $z_{k}<z_{m}$ for any $k<m$, the second summation strictly exceeds the first one, and thus the sign of marginal repayment is strictly positive.

We have shown that the marginal repayment of either a standard debt contract or an outside equity contract is strictly positive, and thus both types of contract incentivize suboptimal effort. It remains to address whether one of the two contracts is preferable, if both yield the same expected repayment. To this end, Innes (1990) provides a general result that also holds in our discrete setting and that we record as the monotonic-contracts hypothesis as follows:

Proposition 2 (Monotonic-contracts hypothesis) In the class of monotonic contracts, the standard-debt contract dominates any other repayment-equivalent monotonic contract, e.g., outside equity, in terms of entrepreneurial profit and allocative efficiency, due to a smaller deviation from first-best effort.

Proof omitted.

\section{Experimental design}

\subsection{Model parameterization, treatments, and theoretical predictions}

In the experiment, we implement the model introduced in Sect. 2 with three states and linear probability functions. The project revenues and probability functions required for states 1, 2, and 3 to occur are as follows:

$$
\begin{array}{ll}
z_{1}=500 \mathrm{ECU} & \text { with } p_{1}(x)=0.6-0.6 \frac{x}{100}, \\
z_{2}=9,000 \mathrm{ECU} & \text { with } p_{2}(x)=0.4, \\
z_{3}=10,000 \mathrm{ECU} & \text { with } p_{3}(x)=0.6 \frac{x}{100},
\end{array}
$$

where effort $x \in[0,100]$. Through increasing effort, probability is shifted from the low project return of 500 ECU to the high project return of 10,000 ECU. This can be thought of as probability mass being shifted from the low to the intermediate return by the same magnitude as from the intermediate to the high return.

The entrepreneur faces a cost of effort of $c(x)=0.5 x^{2}$. The start-up investment of the project is fixed at $\Gamma=3,120 \mathrm{ECU}$. The rate of return required by an outside lender to finance the project is $r=0.25$.

We investigate eight treatments, which we divide into four basic treatments and four extension treatments. The basic treatments provide the building blocks for our discussion of the incentive effects of contract structures, specifically, the nonmonotonic contract, the standard debt contract, the equity contract, and the norepayment contract. They differ in the repayment contract only. The extension treatments serve to extend the discussion to changes in the environment beyond the repayment contract while controlling for selected aspects. 
Table 1 Repayment contracts by basic treatment

\begin{tabular}{|c|c|c|c|c|}
\hline Repayment/Treatment & $\mathrm{SDC}$ & NMC & NoRepay & EQUI $(72.5 \%)$ \\
\hline$t_{1}\left(z_{1}=500\right)$ & $500.00 \mathrm{ECU}$ & $500.00 \mathrm{ECU}$ & $0.00 \mathrm{ECU}$ & $362.50 \mathrm{ECU}$ \\
\hline$t_{2}\left(z_{2}=9000\right)$ & 7,383.30 ECU & $9,000.00 \mathrm{ECU}$ & $0.00 \mathrm{ECU}$ & $6,525.00 \mathrm{ECU}$ \\
\hline$t_{3}\left(z_{3}=10000\right)$ & 7,383.30 ECU & $500.00 \mathrm{ECU}$ & $0.00 \mathrm{ECU}$ & $7,250.00 \mathrm{ECU}$ \\
\hline Effort prediction & 15.7 & 57.0 & 57.0 & 15.7 \\
\hline Total surplus & $1,551.66 \mathrm{ECU}$ & $2,404.50 \mathrm{ECU}$ & $2,404.50 \mathrm{ECU}$ & $1,551.66 \mathrm{ECU}$ \\
\hline Exp. Repayment & 3,900.00 ECU & 3,900.00 ECU & $0.00 \mathrm{ECU}$ & $3,476.30 \mathrm{ECU}$ \\
\hline Entrepreneur's EU & $769.93 \mathrm{ECU}$ & $1,624.50 \mathrm{ECU}$ & $2,404.50 \mathrm{ECU}$ & 1,195.35 ECU \\
\hline Investor's EU & $780.00 \mathrm{ECU}$ & $780.00 \mathrm{ECU}$ & $0.00 \mathrm{ECU}$ & $356.20 \mathrm{ECU}$ \\
\hline
\end{tabular}

To minimize confounding effects that could arise from social preferences or strategic uncertainty, we use an individual-choice experiment where incentive structures are set exogenously by the experimenter and are not affected by the actual choice behavior of subjects in the experiment. This aspect of our design captures the anonymous setting characteristic of many financial markets, as funding contracts are frequently offered through financial institutions such as banks, where social preferences seem less relevant. ${ }^{8}$ We refer to treatments by the name of the implemented contract structure, as this is the main treatment variable and the only treatment variable that changes in the basic treatments. The specifications of the exogenously chosen repayment contracts are as follows: As a benchmark, we run a self-financing treatment (NoRepay), in which there is no repayment at all. In addition, we study three standard-debt-contract conditions (SDC, SDC2, SDC-OS), two non-monotoniccontract conditions (NMC, NMC-R), and two equity conditions (EQUI, EQUI2), in each of which subjects are exposed to the indicated type of repayment contract. Table 1 summarizes all repayment contracts used in the basic treatments, and Table 2 details the contracts used in the extension treatments.

First, consider the repayment contracts of the basic treatments given in Table 1. The required expected repayment to the lender, $(1+r) \Gamma$, determines the statecontingent repayments under the standard debt contract, SDC, and under the nonmonotonic contract, NMC. These two contracts each lead to the same expected repayment of 3,900 ECU. In contrast, the state-contingent repayments in treatment EQUI are chosen so that the effort prediction equals the effort prediction prevailing in treatment SDC, namely 15.7. This requires that the state-independent equity share is $72.5 \%$. Evidently, the standard debt contract condition, SDC, and the equity contract condition, EQUI, lead to a loss in total surplus, and the entrepreneur's payoff is substantially smaller. If the standard debt contract of treatment SDC is replaced by the repayment-equivalent non-monotonic contract of treatment NMC, total surplus increases by $55 \%$, while the surplus accruing to the entrepreneur more than doubles. ${ }^{9}$

\footnotetext{
${ }^{8}$ Reiß and Wolff (2013) endogenize the selection of repayment contracts, studying the structures of subjectselected repayment contracts and their effects on entrepreneurial effort.

${ }^{9}$ The exact numbers are $54.96 \%$ and $110.99 \%$, respectively.
} 
Table 2 Repayment contracts by extension treatments

\begin{tabular}{|c|c|c|c|c|c|}
\hline Repayment/Treatment & NMC-R & SDC2 & & EQUI2 (80\%) & SDC-OS \\
\hline$t_{1}\left(z_{1}=500\right)$ & $500.00 \mathrm{ECU}$ & $500.00 \mathrm{ECU}$ & & $400.00 \mathrm{ECU}$ & $500.00 \mathrm{ECU}$ \\
\hline$t_{2}\left(z_{2}=9000\right)$ & $9,000.00 \mathrm{ECU}$ & $6,449.40 \mathrm{ECU}$ & & 7,200.00 ECU & 7,383.30 ECU \\
\hline$t_{3}\left(z_{3}=10000\right)$ & $500.00 \mathrm{ECU}$ & $6,449.40 \mathrm{ECU}$ & & $8,000.00 \mathrm{ECU}$ & 7,383.30 ECU \\
\hline Effort prediction & 57.0 & 21.3 & & 11.4 & 15.7 \\
\hline Total surplus & 2,404.50 ECU & 1,767.38 ECU & & $1,364.82 \mathrm{ECU}$ & $1,551.66 \mathrm{ECU}$ \\
\hline Exp. Repayment & $500.00 \mathrm{ECU}$ & 3,640.22 ECU & $\simeq$ & 3,639.84 ECU & 3,900.00 ECU \\
\hline Entrepreneur's EU & $5,024.50 \mathrm{ECU}$ & $1,247.16 \mathrm{ECU}$ & & $844.98 \mathrm{ECU}$ & $769.93 \mathrm{ECU}$ \\
\hline Investor’s EU & $-2,620.00 \mathrm{ECU}$ & $520.22 \mathrm{ECU}$ & $\simeq$ & $519.84 \mathrm{ECU}$ & $780.00 \mathrm{ECU}$ \\
\hline
\end{tabular}

Second, consider the repayment contracts used in the extension treatments as given in Table 2. The non-monotonic contract used in treatment NMC-R is identical to the one used in treatment NMC, but both treatments differ in the way realized project return states are reported to the computerized investor. While there is automatic and accurate reporting of the realized return state in treatment NMC, entrepreneurs themselves report the realized return state with no verification in treatment NMC-R when state reporting is relevant, i.e., in cases of a medium return or a high return state. This means that entrepreneurs can falsely report a high return state when a medium return state is realized to decrease the state contingent repayment from 9,000 ECU to $500 \mathrm{ECU}$ in NMC-R. Thus, an entrepreneur with self-regarding monetary preferences finds it optimal to falsely report a high return state whenever a medium return state is realized so that the repayment is always $500 \mathrm{ECU}$ regardless of the realized state. As a result, treatment NMC-R allows the entrepreneur to significantly increase the expected payoff at the expense of the investor through inaccurate state reporting.

Treatments SDC2 and EQUI2 are designed so that the expected repayments to the investor are the same in the two cases. Although there is a minute difference of $0.38 \mathrm{ECU}$ between the expected repayments, we neglect it and regard the expected repayments in either treatment as sufficiently close to be essentially the same. Importantly, the difference in contract structures implies substantially different effort predictions which is reflected in a large predicted loss of total surplus in EQUI2 as compared to SDC2. Finally, treatment SDC-OS is identical to basic treatment SDC, except that SDC-OS implements subjects' choice of effort level as a one-shot decision with no repetition and high-powered incentives, while there are 15 rounds with feedback in treatment SDC and the other treatments.

Behavior in the laboratory that deviates from our theory-based predictions, which assume risk-neutrality, may be attributable to the effects of individual risk preferences such as various degrees of risk-aversion. To address this concern, we reduce the risk in subjects' payoffs by paying them the average payoff over 50 different projects, with outcomes determined by independent draws from the probability distribution determined by effort choice instead of using the payoff realized for a single project. This method was successfully introduced by Kirchkamp et al. (2008) in an auction setting and further explored in an individual choice setting by Niemeyer et al. (2013). 


\subsection{Procedures and other details}

The experiment was programmed using z-tree (Fischbacher 2007). We conducted two experimental sessions for each treatment. The first session for each of the treatments, NoRepay, SDC, NMC, and EQUI, was conducted at the Erfurt Laboratory for Experimental Economics (eLab), and the second was run at the Lakelab of the University of Konstanz. Both experimental sessions for the treatments SDC-OS, NMC-R, SDC2, and EQUI2 were run at the Behavioral and Experimental Economics Laboratory at Maastricht University (BEElab). Twelve subjects were recruited for each session, using ORSEE (Greiner 2004) at all three locations. In the second experimental session of treatment EQUI, there were eleven rather than twelve participating subjects, owing to no-shows. Therefore, in total, $8 \times 2 \times 12-1=192-1=191$ subjects participated in the study, with no subject participating in more than one session. As we ran two sessions for each treatment, we obtained 24 independent observations per treatment, except for treatment EQUI, where we obtained 23 independent observations.

On the day of the experiment, subjects were welcomed and randomly assigned to private cabins. Written instructions were handed to them before being read aloud by the experimenter. Subsequently, subjects entered their cubicles and had some time to go over the instructions again and ask any questions they might have. Questions were answered individually.

Profit obtained by a subject in any round of the experiment was added to the subject's capital balance and any loss was subtracted. At the end of the experiment, capital balances were converted into EUR and paid to subjects in cash. In principle, it is possible for subjects to go bankrupt by repeatedly choosing excessively high effort levels, with the high costs of effort causing losses to accumulate. To prevent bias of chosen effort levels by limited liability considerations, each subject was given an initial endowment of 12,500 ECU. ${ }^{10}$ The endowment allowed subjects to survive several rounds of the experiment, with a maximum effort of 100, at a cost in effort of 5,000 ECU. The instructions informed subjects that they would be removed from the experiment if their balance dropped below 2,500 ECU. ${ }^{11}$ We did not expect to observe any cases of bankruptcy; however, it occurred once in treatment EQUI, where a subject was bankrupt in round 6 after selecting the effort levels of 90, 85, 100, 100, 95 , and 100 in rounds $1-6$. We removed this observation from the data set used for analysis, as the observation was incomplete.

Subjects played 15 rounds of the game, except in the case of treatment SDCOS, which involved one round of decision making, and were paid according to their individual performances. The experimental sessions lasted for one hour or less, with average earnings of $€ 9.65(\approx$ US\$ 12.50) for the experiments at the eLab, $€ 9.91$ $(\approx$ US\$ 12.84) for the experiments at the Lakelab and $€ 13.33(\approx$ US\$17.27) at the BEElab. ${ }^{12}$ Payments were settled individually to ensure subjects' anonymity.

\footnotetext{
${ }^{10}$ Exceptions were the NoRepay treatment and the one-shot treatment SDC-OS with different conversion rates, where the endowments were set to 100,000 ECU and 3,000 ECU, respectively.

${ }^{11}$ In treatment NoRepay, the threshold was 20,000 ECU and in the one-shot treatment SDC-OS, bankruptcy procedures were irrelevant and not mentioned in the instructions.

${ }^{12}$ Average earnings in Maastricht were higher than in Erfurt and Konstanz due to treatment differences that allowed for higher earnings, e.g., with false state reporting in treatment NMC-R.
} 


\subsection{Testable hypotheses}

We derive the hypotheses that we test in the experiment from the theoretical predictions summarized in Tables 1 and 2. At the least demanding level, we expect that effort choices are systematically influenced by the contract conditions. In particular, we hypothesize that observed behavior is qualitatively consistent with the comparative statics of changing the funding contract. This leads to our first and most basic hypothesis:

Hypothesis 1 Observed effort choices are influenced by contract conditions and share the ordinal rank across contract conditions with the theoretical ranking prediction:

$$
x^{\text {NoRepay }} \approx x^{\mathrm{NMC}}>x^{\mathrm{SDC}} \approx x^{\mathrm{EQUI}} .
$$

Hypothesis 1 is weak in the sense that it is a qualitative hypothesis that disregards the quantitative nature of the theoretical point predictions. As the precise optimal effort values allow us to also predict behavior quantitatively, we strengthen the first hypothesis by additionally hypothesizing that behavior is also consistent with the point predictions:

Hypothesis 2a Observed effort choices on average match the theoretical point predictions of effort.

A particular strength of the model is its parsimony. It provides a single equation, (1), that predicts effort levels for any repayment contract. Although Hypothesis 2a relates to the optimal effort equation, it pertains to the comparison of observed effort to predicted effort for each contract condition separately. This allows for some flexibility, as the point prediction for a given contract condition may fit the data for some repayment contract better than for other repayment contracts. To strengthen our hypothesis on the theory's predictive power, Hypothesis $2 \mathrm{~b}$ proposes that the optimal effort prediction simultaneously holds for all repayment contracts in the basic treatments:

Hypothesis 2b The optimal effort function (1) explains observed effort choices well in all basic contract conditions simultaneously.

The non-monotonic-contracts hypothesis compares allocative efficiency and profits obtained under the non-monotonic contract NMC to those obtained under the standard debt contract SDC, theoretically yielding the same expected investor repayment.

Hypothesis 3 (Non-monotonic-contracts hypothesis) Allocative efficiency and entrepreneurial profits are higher under the non-monotonic contract NMC than under the standard debt contract SDC.

Innes (1990) has shown that the standard debt contract dominates any other repayment-neutral contract in the class of monotonic contracts. We summarize this result as the monotonic-contracts hypothesis: 
Hypothesis 4 (Monotonic-contracts hypothesis) Effort, allocative efficiency, and entrepreneurial profits are higher under the standard debt contract SDC2 than under the repayment-equivalent equity contract EQUI2.

The state-contingency of repayments under the non-monotonic contract is crucial for its success in incentivizing first-best effort. At the same time, this key characteristic provides strong incentives to misreport states in order to manipulate the repayment. This leads to practical disadvantages of the non-monotonic contract whenever there are opportunities for manipulation. We test for the misrepresentation of states with treatment NMC-R, where subject entrepreneurs themselves report, without state verification, the project outcomes determining investor repayments. Here we advance the hypothesis that subjects tend to accurately report realized payoff states in relevant cases, though there is considerable evidence that subjects misrepresent states if it is to their monetary advantage to do so: ${ }^{13}$

Hypothesis 5 (No-misrepresentation hypothesis) The number of reported medium return states is equal to the number of realized medium return states.

In light of our experimental results, we will argue that experience of contract incentives matters. Experience with contract incentives accumulates over the course of the experiment. As the cash payoffs of our subjects accumulate over the fifteen rounds of the experiment, subjects are exposed to relatively low monetary incentives in a given round. This raises the question of whether it is subjects' lack of experience at the beginning of the experiment that explains suboptimal choices or whether subjects employ an inexpensive experimentation strategy to find their way to the optimum instead of reasoning about contract incentives ex ante. To control for the latter, we compare the effort levels observed in a one-shot treatment, where only ex ante reasoning about contract incentives matters, to the first-round choices in an equivalent multiple-rounds treatment, expecting to find no differences:

Hypothesis 6 (Experience-matters hypothesis) Deviations in effort level from the predicted effort level in the first round of fifteen rounds observed in treatment SDC are similar to these observed in the single-round treatment SDC-OS.

\section{Experimental results}

First, we investigate whether incentives matter, and address the experience-matters hypothesis, using the results of the control treatment SDC-OS. We then examine the non-monotonic-contracts hypothesis and the no-misrepresentation hypothesis. Finally, we analyze the monotonic-contracts hypothesis.

\subsection{Effects of funding contracts on effort choice}

Let us begin by addressing the fundamental question of whether funding contracts affect behavior at all, and if so, to what extent this behavior is consistent with theoretical

\footnotetext{
${ }^{13}$ See, e.g., Fischbacher and Heusi (2013) and Mazar et al. (2008).
} 


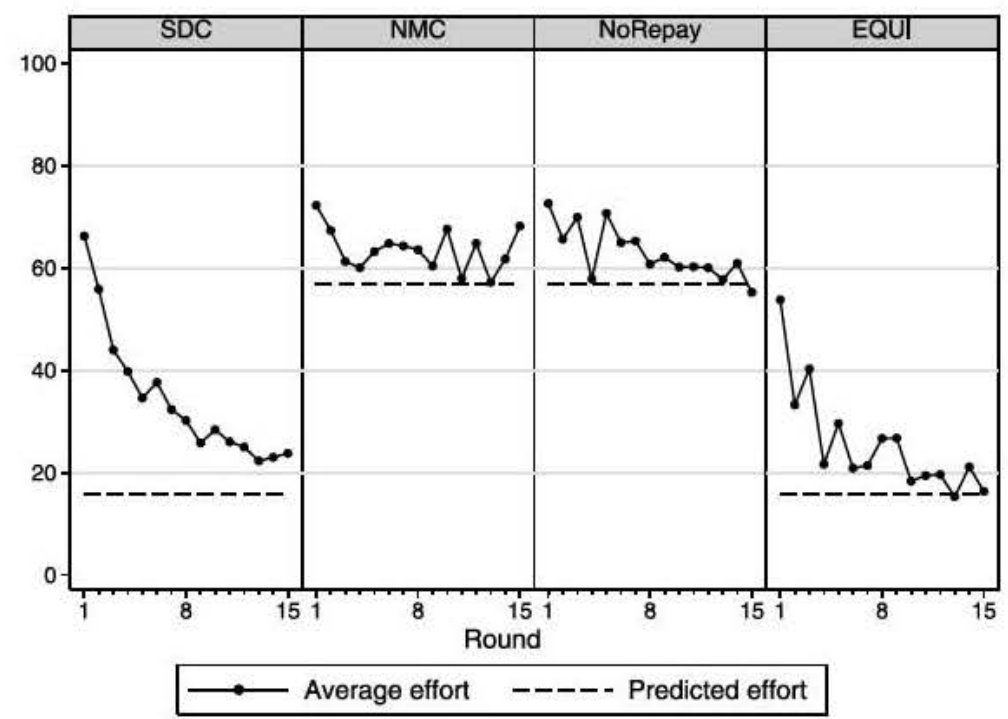

The figure depicts average effort (solid lines) over rounds of the experiment under the standard debt contract (left), under the non-monotonic contract (second from left), under no-repayment (second from right), and under outside equity (right). The theoretical effort prediction is indicated by dashed lines.

Fig. 1 Average effort and predicted effort by round

predictions. In the experiment, any incentive effect of funding contracts should be reflected directly in observed effort levels. Figure 1 depicts average effort by treatment and by round. The patterns of the average effort paths across contract types show that funding contracts embody incentives that strongly affect behavior. Hypothesis 1 , on comparative statics, appears to be largely confirmed. The paths of average effort, $\left\{\bar{x}_{t}, t=1, \ldots, 15\right\}$, appear roughly similar when they are expected to be similar, $\bar{x}_{t}^{\mathrm{SDC}} \approx \bar{x}_{t}^{\mathrm{EQUI}}$ and $\bar{x}_{t}^{\mathrm{NMC}} \approx \bar{x}_{t}^{\text {NoRep }}$ and seem to differ in the hypothesized direction when they are expected to differ, $\bar{x}_{t}^{\mathrm{NMC}}, \bar{x}_{t}^{\mathrm{NoRep}}>\bar{x}_{t}^{\mathrm{SDC}}, \bar{x}_{t}^{\mathrm{EQUI}}$. This impression is formally confirmed by testing for differences in average effort among any pair of contract conditions, separately for each round, using two-tailed Mann-Whitney- $U$-tests and $t$-tests; see Table 3 for a summary. The table shows the number of significant and insignificant differences in average effort that we find for each pair of contract conditions. The test results indicate that we observe significant differences in average effort in almost all rounds for all cases where theory predicts differences in average effort between contract conditions. Similarly, for all cases where theory predicts that average effort should not differ across contract conditions, we observe in nearly all rounds no significant differences, except for the treatment comparison of SDC and EQUI where we observe significant differences more often than predicted in the first half of the experiment, but not at the end of the experiment. We further analyze this issue with a regression that accounts for intra-subject correlations. The regression results confirm the absence of statistical differences between SDC and EQUI at the end of the experiment, see Electronic Supplementary Material. Hence, we find support for Hypothesis 1. 
Table 3 Summary of Mann-Whitney $U$-tests and $t$-tests comparing effort across contract conditions

\begin{tabular}{|c|c|c|c|}
\hline Contract condition & NMC & NoRepay & EQUI \\
\hline SDC & $x^{\mathrm{SDC}}<x^{\mathrm{NMC}}$ & $x^{\text {SDC }}<x^{\text {NoRepay }}$ & $x^{\mathrm{SDC}}=x^{\mathrm{EQUI}}$ \\
\hline No. of sign. diffs. (MWU) & $14(p \leq 0.069)$ & $13(p \leq 0.018)$ & $5(p \leq 0.080)$ \\
\hline No. of insign. diffs. (MWU) & $1(p \geq 0.438)$ & $2(p \geq 0.112)$ & $10(p \geq 0.1004)$ \\
\hline No. of sign. diffs. ( $t$-test) & $13(p \leq 0.021)$ & $13(p \leq 0.015)$ & $8(p \leq 0.097)$ \\
\hline No. of insign. diffs. ( $t$-test) & $2(p \geq 0.113)$ & $2(p \geq 0.207)$ & $7(p \geq 0.235)$ \\
\hline Round average (rds. 1-15, MWU) & $p=0.000$ & $p=0.000$ & $p=0.015$ \\
\hline Round average (rds. $1-15, t$-test) & $p=0.000$ & $p=0.000$ & $p=0.007$ \\
\hline Round average (rds. 9-15, MWU) & $p=0.000$ & $p=0.000$ & $p=0.113$ \\
\hline Round average (rds. 9-15, $t$-test) & $p=0.000$ & $p=0.000$ & $p=0.091$ \\
\hline NMC & & $x^{\mathrm{NMC}}=x^{\text {NoRepay }}$ & $x^{\mathrm{NMC}}>x^{\mathrm{EQUI}}$ \\
\hline No. of sign. diffs. (MWU) & & 0 & $15(p \leq 0.039)$ \\
\hline No. of insign. diffs. (MWU) & & $15(p \geq 0.101)$ & 0 \\
\hline No. of sign. diffs. ( $t$-test) & & $1(p=0.057)$ & $15(p \leq 0.015)$ \\
\hline No. of insign. diffs. ( $t$-test) & & $14(p \geq 0.134)$ & 0 \\
\hline Round average (rds. 1-15, MWU) & & $p=0.688$ & $p=0.000$ \\
\hline Round average (rds. $1-15, t$-test) & & $p=0.850$ & $p=0.000$ \\
\hline Round average (rds. 9-15, MWU) & & $p=0.757$ & $p=0.000$ \\
\hline Round average (rds. 9-15, $t$-test) & & $p=0.504$ & $p=0.000$ \\
\hline NoRepay & & & $x^{\text {NoRepay }}>x^{\text {EQUI }}$ \\
\hline No. of sign. diffs. (MWU) & & & $15(p \leq 0.022)$ \\
\hline No. of insign. diffs. (MWU) & & & 0 \\
\hline No. of sign. diffs. $(t$-test $)$ & & & $15(p \leq 0.017)$ \\
\hline No. of insign. diffs. ( $t$-test) & & & 0 \\
\hline Round average (rds. 1-15, MWU) & & & $p=0.000$ \\
\hline Round average (rds. $1-15, t$-test) & & & $p=0.000$ \\
\hline Round average (rds. 9-15, MWU) & & & $p=0.000$ \\
\hline Round average (rds. 9-15, $t$-test) & & & $p=0.000$ \\
\hline
\end{tabular}

The table reports, for any pair of treatments, the results of roundwise Mann-Whitney $U$-tests and $t$-tests. The null hypothesis is that there is no difference in the central locations of effort or average effort observed in the paired treatments. The alternative hypothesis is that such differences exist. For any comparison of treatments, the theoretical comparative statics effort prediction and the number of significant and insignificant differences among the 15 roundwise tests are reported together with the highest and lowest obtained $p$-values in parentheses. In addition, the $p$-value for the test on the round averages is reported

Comparisons of the paths of average effort to the theoretical predictions, as indicated by the dashed lines in Fig. 1, show, however, that observed behavior is much richer than predicted by theory. For example, in contrast to the static prediction for the contract condition SDC, $x^{\text {SDC }}=15.7$, observed average effort changes considerably over the course of the experiment, as seen in the left panel of the figure. The graph for the contract condition SDC (left panel) shows, nevertheless, that the static prediction turns out to be rather useful, as it attracts observed average effort over time. Overall, 
the standard debt contract leads subjects to choose inefficiently low levels of effort most of the time, as predicted by theory, but not at the beginning rounds of the experiment: In the first two rounds of the experiment, average effort is in the vicinity of the first-best level of $x^{*}=57$. In the first round, average effort overshoots the first-best level (two-tailed $t$-test, $p=0.042$ ), and in the second round, there is no significant difference $(p=0.802)$, which is inconsistent with the theoretical prediction for the SDC condition. For all of the remaining 13 rounds, the $t$-test indicates significant differences between average effort and first-best effort (all $p<0.018) .^{14}$

Comparisons of the data to the theoretical point prediction of inefficient effort, $x^{\mathrm{SDC}}=15.7$, show that average effort differs greatly from this prediction, except for the second half of the experiment: According to the sign test, effort is not significantly different from the predicted effort levels in rounds 8 to 15 (two-tailed, $p \geq 0.152$ ), while the test identifies significant differences for all earlier rounds $(p<0.024)$. The $t$-test, however, finds significant differences in all rounds (two-tailed, $p \leq 0.028$ ) suggesting that observed convergence is imperfect and slightly bounded away from the point prediction. ${ }^{15}$

Next, we consider the outside equity contracts in more detail. This type of contract is designed to induce the same level of effort as the standard debt contract, $x^{\mathrm{EQ}}=x^{\mathrm{SDC}}=15.7$. Though average effort observed in the outside equity condition (right panel) evolves very similarly to that observed in the SDC condition (left panel), one apparent subtle difference between the paths of average effort is that convergence towards the theoretically predicted effort level is slightly faster under the outside equity contract. This is consistent with the results of roundwise comparisons of average effort with predicted effort levels, as deviations from predicted levels fade away in later rounds under the SDC condition. In the EQUI condition, the $t$-test finds significant differences in five rounds, in the first three rounds and in rounds 5 and 9 (two-tailed, $p<0.039$ for rounds 1-3, 5, 9 and $p>0.105$ for all other rounds), while it finds a significant difference in each of the rounds in the SDC condition. ${ }^{16}$

In contrast to the convergence of average behavior under the standard debt contract and under the outside equity contract, there is neither convergent nor divergent behavior under the non-monotonic contract or under the no-repayment contract. In the treatment conditions NMC and NoRepay, first-round average effort is close to the theoretical prediction of $x^{\mathrm{NMC}}=x^{\text {NoRepay }}=57$ and appears to fluctuate in the neighborhood of the theoretical prediction over time, as seen in Fig. 1. In fact, roundwise comparisons of average effort to the predicted levels do not suggest a systematic trend over time. Only a few significant differences appear to be arbitrarily distributed over the course of the experiment in either treatment. Specifically, the $t$-test reveals significant differences in five rounds $(1-2,6,10$, and $15, p<0.07)$ in condition NMC

\footnotetext{
${ }^{14}$ Similarly, the sign test reveals significant differences between observed median effort and first-best effort in rounds 3 to 15 (two-tailed, $p<0.023$ ); for the remaining two rounds at the beginning of the experiment, observed differences are insignificant in round $2(p=0.540)$ and significant in round $1(p=0.007)$.

${ }^{15}$ For a more detailed exploration of learning effects, see Sect. 4.3 in the working paper version Reiß and Wolff (2012)

${ }^{16}$ The sign test indicates significant differences in rounds $1-3,9$, and 14 (two-tailed, $p<0.053$ ) in the EQUI treatment and in the first seven rounds $(p<0.024)$ in the SDC treatment.
} 
and significant differences in four rounds $(1,3,5$, and $7, p<0.062)$ in condition NoRepay. ${ }^{17}$

Result 1 Funding contracts strongly influence the choice of effort in a way that is consistent with the comparative statics predictions, except in the early rounds of the experiment (support for Hypothesis 1). Behavior adjusts to the theoretical point predictions through repeated exposure to incentives over time (partial support for Hypothesis $2 a$ ).

To quantify the extent to which the incentives of funding contracts influence effort choice, once incentives have been absorbed, we estimate the first-order condition (1), using data from the second half of the experiment, i.e., rounds 9-15. In our parameterization, the first-order condition can be explicitly solved for optimal effort and simplifies to

$$
x^{*}=57-\frac{6\left(t_{3}-t_{1}\right)}{1,000}
$$

First, we estimate ${ }^{18}$ the unrestricted model

$$
x_{i t}=\beta_{0}+\beta_{1} \widetilde{t}_{1}+\beta_{2} \widetilde{t}_{2}+\beta_{3} \widetilde{t}_{3}+u_{i t}
$$

The dependent variable $x_{i t}$ is the effort level chosen by participant $i$ in round $t, \widetilde{t}_{s}$ is the repayment in state $s$ measured in thousands of ECU (i.e., $\tilde{t}_{s}=t_{s} / 1,000$ ), and $u_{i t}$ is the residual. The unrestricted model does not impose any restrictions derived from theory on the specification, apart from the linearity assumption. This allows us to explore whether repayment in state $2, t_{2}$, though theoretically irrelevant, affects effort choice and to examine whether repayments in states 1 and 3 have similarly strong effects on behavior.

If observed behavior is fully consistent with theory, then we would expect to estimate coefficients indicating that the optimal effort function (2) is reproduced by specification (3), i.e., $\widehat{\beta_{0}}=57, \widehat{\beta_{1}}=6, \widehat{\beta_{2}}$ not significantly different from zero, and $\widehat{\beta_{3}}=-6$. Table 4 presents regression results that are broadly consistent with theory. Although the estimates reproduce essential features of the optimal effort function, the joint hypothesis that the estimated coefficients satisfy the theoretical point predictions is rejected at the $1 \%$ significance level. ${ }^{19}$

In our parameterization, the additional repayment to the investor that arise if the entrepreneur devotes one more unit of effort to the project, i.e., the marginal repayment, is constant for any repayment contract and given by

$$
\mathrm{MR}=\frac{6\left(t_{3}-t_{1}\right)}{1,000}
$$

\footnotetext{
${ }^{17}$ The sign test finds significant differences in rounds $1-2$ and $15(p<0.064)$ in the NMC condition and significant differences in four rounds $(1,5,7$, and $12, p \leq 0.064)$ in the NoRepay condition.

${ }^{18}$ We estimate this and the next model using OLS, such that the computation of standard errors takes into account that observations of the same individual might be correlated across time (Rogers 1993).

${ }^{19} \mathrm{An} F$-test of the joint hypothesis (I) $\widehat{\beta_{0}}=57$, (II) $\widehat{\beta_{1}}=-\widehat{\beta_{3}}$, (III) $\widehat{\beta}_{1}=6$, (IV) $\widehat{\beta_{2}}=0$ with $F_{4,93}=5.94$ yields $p=0.0003$.
} 
Table 4 Estimation results of (3) and (4)

\begin{tabular}{|c|c|c|c|c|c|c|}
\hline & Coefficient & Robust $\sigma$ & $t$ & $p$-value & $95 \%$ conf & Interval \\
\hline \multicolumn{7}{|c|}{ (I) Unrestricted model (3) } \\
\hline (Intercept) & 59.48 & 3.379 & 17.60 & 0.000 & 52.77 & 66.19 \\
\hline$\tilde{t}_{1}$ & 62.03 & 34.102 & 1.82 & 0.072 & -5.69 & 129.75 \\
\hline$\tilde{t}_{2}$ & -2.77 & 2.017 & -1.37 & 0.174 & -6.77 & 1.24 \\
\hline$\tilde{t}_{3}$ & -6.12 & 0.439 & -13.93 & 0.000 & -6.99 & -5.25 \\
\hline \multicolumn{7}{|c|}{ No. of obs: 658, No. of clusters: $94, R^{2}=0.5060$} \\
\hline \multicolumn{7}{|c|}{ (II) Restricted model (4) } \\
\hline (Intercept) & 61.01 & 2.251 & 27.10 & 0.000 & 56.54 & 65.48 \\
\hline Marg. Repayment & 0.94 & 0.067 & 14.09 & 0.000 & 0.80 & 1.07 \\
\hline
\end{tabular}

Note: State-contingent repayments are normalized such that $\widetilde{t}_{s}=t_{s} / 1,000$. Estimation results are computed by OLS with robust standard errors using data from the second half of the experiment, rounds $9-15$

The marginal repayment depends on the funding contract through the repayments in states 1 and 3 only. Comparison of the marginal repayment MR to the optimal effort function (2) shows that optimal effort is given simply by first-best effort, $x^{*}=57$, minus marginal repayment. To quantify the effect of a funding contract's marginal repayment on effort, we regress observed effort on the marginal repayment, using the following restricted specification:

$$
x_{i t}=\gamma_{0}-\gamma_{1} 6\left(\tilde{t}_{3}-\tilde{t}_{1}\right)+u_{i t}
$$

In this regression equation, the coefficient $\gamma_{1}$ indicates the effect of the marginal repayment on effort. Theoretically, we expect to find an estimate of $\widehat{\gamma}_{1}=1$. Any positive estimate, $\widehat{\gamma}_{1}>0$, would indicate that a reduction in effort would be correlated with such changes in the repayment contract that imply greater repayment if the entrepreneur exerts additional effort. An estimate of $\widehat{\gamma}_{1}>1$ would imply that observed effort would respond excessively to contractual changes associated with changes in marginal repayment. In this case, a replacement of the standard debt contract (with strictly positive marginal repayment) by a non-monotonic contract (with zero marginal repayment) would increase effort by an amount greater than the amount theoretically predicted. Table 4 reports the estimation results. It turns out that the coefficient on marginal repayment is not significantly different from one (two-tailed $t$-test, $p=0.338$ ). Therefore, on average, marginal repayment captures the incentives provided by funding contracts on effort choice, as theoretically predicted. We summarize our results on observed effort levels as compared to optimal choice as follows:

Result 2 Observed average behavior is largely consistent with the theoretical point predictions given by the optimal effort choice function (1), once sufficient experience accumulates (partial support for Hypothesis $2 b$ ). 
Table 5 Effort choices in treatment SDC-OS and in the first round of treatment SDC

\begin{tabular}{llll}
\hline Treatment/Statistic & Average & Median & Std. Dev. \\
\hline SDC (first round) & 66.2 & 67.0 & 21.012 \\
SDC-OS & 65.1 & 72.5 & 26.694 \\
SDC effort prediction & 15.7 & & \\
\hline
\end{tabular}

\subsection{Experience-matters hypothesis: control treatment SDC-OS}

In the preceding subsection, we interpreted the convergence of average effort towards the optimum, observed over the course of the experiment, as showing that subjects must acquire sufficient experience with contract incentives before incentives take effect. A similar convergence pattern would result, however, if subjects used a trialand-error strategy in selecting their effort levels. In the basic treatments discussed above, the cost of adopting this experimentation strategy at the beginning of the experiment is rather low, as cash earnings accumulate over each of the fifteen rounds. To test the validity of this explanation, we compare first-round choices in the 15rounds-treatment SDC to those in the single-round treatment SDC-OS, where the cash value of one ECU is raised by a factor of fifteen, so that monetary incentives are high-powered in the latter treatment.

Table 5 shows that the average effort level in treatment SDC-OS, 65.1, exceeds the predicted value of 15.7. Formal testing reveals that this difference is statistically significant (two-tailed $t$-test, $p=0.000$ ). ${ }^{20}$ Further, the descriptive data show that the average effort in treatment SDC-OS is virtually equal to the average effort observed in the first round of treatment SDC; the difference is insignificant (two-tailed $t$-test, $p=0.874){ }^{21}$ Thus, we rule out the possibility that subjects follow the trial-anderror-strategy, concluding that the actual experience of contract incentives matters, experience that cannot be substituted for by raising monetary incentives to promote ex ante reasoning about contract incentives.

Result 3 Effort choices in the one-shot, high-powered incentives treatment SDC-OS are similar to those observed in the first round of treatment SDC (support for Hypothesis 6).

\subsection{Non-monotonic-contracts hypothesis}

In this section, we take a closer look at the non-monotonic-contracts hypothesis. Would the replacement of a standard debt contract by a repayment-equivalent nonmonotonic contract reduce efficiency losses and increase entrepreneurial income as predicted? Figure 2 shows the average incomes obtained under both contracts for each round. It is easy to see that entrepreneurial income in the NMC condition greatly ex-

\footnotetext{
${ }^{20}$ Two-tailed sign test, $p=0.000$.

${ }^{21}$ Two-tailed MWU-test, $p=0.984$.
} 


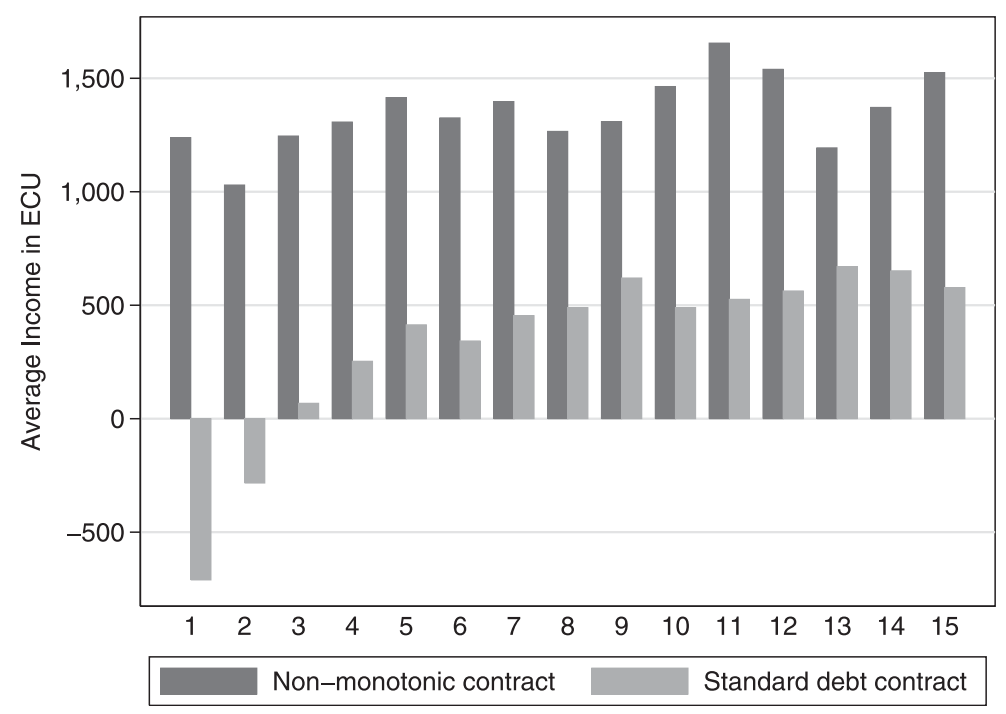

Fig. 2 Average round income of entrepreneurs under NMC and SDC

ceeds that in the SDC condition. Using data from the second half of the experiment, rounds $9-15$, we find that NMC income exceeds SDC income by $150 \% .^{22}$

Result 4 Observed entrepreneurial income (net of the cost of effort and repayment) under the non-monotonic contract is on average $150 \%$ higher than that under the standard debt contract (support for Hypothesis 3).

Using the two-tailed $t$-test for roundwise comparisons of entrepreneurial income (net of agency costs and repayment) formally confirms that the income difference is highly significant in all fifteen rounds $(p<0.006){ }^{23}$

Figure 3 illustrates how much additional total surplus would have been created in the SDC treatment if, instead of the standard debt contract, a non-monotonic contract had been used. The figure reveals that in the first three rounds of the experiment, there is essentially no welfare disadvantage associated with the standard debt contract. ${ }^{24}$ Clearly, these initial effort levels are suboptimal and yield negative round incomes, as seen in Fig. 2. In the course of the experiment, subjects in the SDC treatment reduce their effort levels toward the individually optimal level. As effort levels in the SDC treatment decrease, the inefficiency of the standard debt contracts increases

\footnotetext{
${ }^{22}$ If earlier rounds are included, non-monotonic contracts perform even better. For example, NMC income tops SDC income by $315 \%$ on average, if data for rounds 3-15 are used.

${ }^{23}$ Similarly, the two-tailed Mann-Whitney $U$ test indicates highly significant income differences in all 15 rounds $(p<0.008)$.

${ }^{24}$ In the first two rounds, SDC welfare is even slightly higher than NMC welfare. This is due to the fact that quite large effort levels are initially chosen in the SDC treatment, similar to those observed in the NMC treatment but somewhat closer to first-best effort, indicated by the horizontal line in the figure.
} 


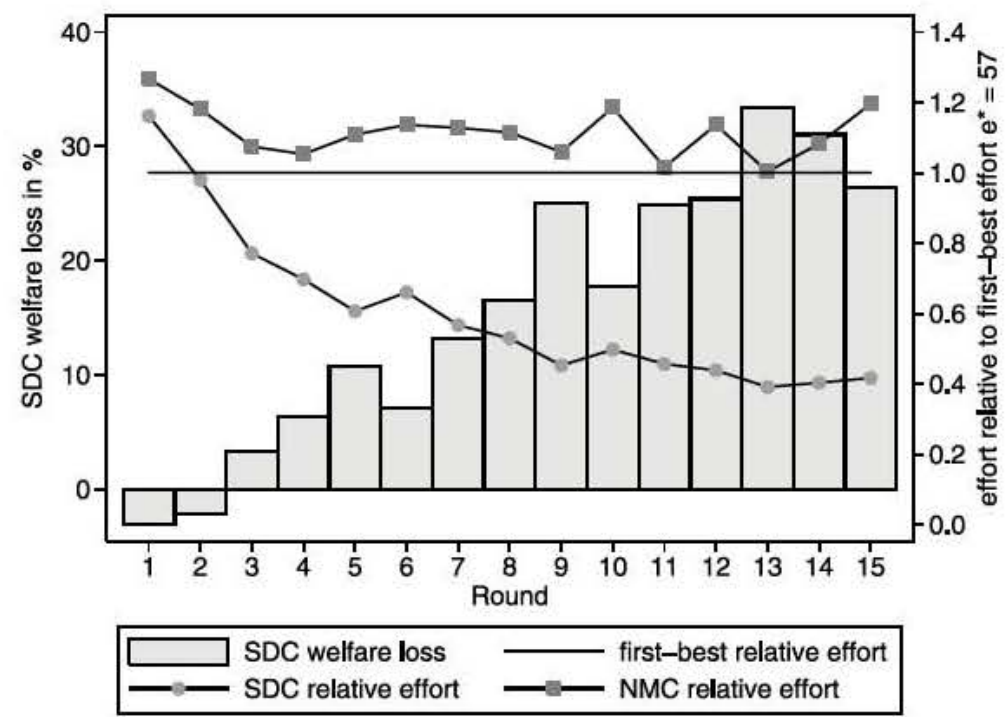

The figure depicts the additional total surplus (in \%) that is created on average if the SDC condition is replaced by the corresponding NMC condition, given average effort levels observed under both contract conditions. This measure of welfare loss is shown as a series of bars (left scale). Furthermore, the figure illustrates average effort observed in both contract conditions relative to first-best effort (right scale). For example, in round 5, NMC average effort exceeds first-best effort by roughly $10 \%$, while SDC average effort falls short by $40 \%$. Finally, the first-best effort benchmark (where total surplus is maximized) is represented by a horizontal line at unity (right scale).

Fig. 3 Welfare loss with a standard debt contract, but eliminable by a non-monotonic contract

sharply. Restricting attention to data from the second half of the experiment (rounds 9-15), where effort levels under SDC have somewhat stabilized (see Fig. 3), we find that use of the non-monotonic contract would have increased total surplus in the SDC treatment by approximately $26 \%$. Total surplus in the NMC treatment is significantly larger than in the SDC treatment in rounds $11-15$ ( $t$-test, $p<0.025$, two-tailed), but not in rounds 9 and $10(p>0.113) .^{25}$

Result 5 Standard debt contracts lead to allocative inefficiencies that can be eliminated by using repayment-equivalent non-monotonic contracts (support for Hypothesis 3).

Our experiment provides strong evidence that non-monotonic contracts dominate standard-debt contracts. ${ }^{26}$ This result, however, obtains in an environment in which there is accurate and costless state verification, ruling out any disagreement between the investor and the entrepreneur about the project's realized return state. It is, however, unclear whether the reported benefits of the non-monotonic contract would be

\footnotetext{
${ }^{25}$ Mann-Whitney $U$ test, two-tailed, for rounds $11-15, p<0.044$; and for rounds 9 and $10, p>0.117$.

${ }^{26}$ Please see Electronic Supplementary Material for a regression analysis confirming the dominance of non-monotonic-contracts in terms of effort, entrepreneurial income, and total surplus.
} 
sustained in the absence of costless state verification. Whenever there are opportunities in real life for an investor or entrepreneur to misrepresent states, such opportunities might be exploited, giving rise to practical disadvantages. In the next subsection we report the results of treatment NMC-R, designed to test whether the availability of entrepreneur-sided state manipulation may erode the participation constraints of parties, as theoretically predicted.

\subsection{No-misrepresentation hypothesis}

The non-monotonic contract relies on accurate reporting of realized states. If there are opportunities for misrepresentation of realized states, then the mutual acceptance of a non-monotonic contract structure by lender and borrower may erode due to incentives for state misreporting and the implied payoff redistribution between the contracting parties. In treatment NMC-R, we test a reduced form of outside borrowing that captures the opportunity for an entrepreneur-sided manipulation of states and associated repayments. Specifically, entrepreneurs can misrepresent a medium return state (with high repayment under NMC-R) as a high return state (with low repayment under NMC-R), and vice versa. Misrepresenting a medium return state by a high return state allows the entrepreneur, in our experiment, to repay 500 ECU instead of 9000 ECU.

Figure 4 shows the evolution of the average number of realized high return states by round. As can be seen, the average number of high return states does not vary much over the course of the experiment, remaining between fifteen and twenty in all rounds. This follows from the average effort level in treatment NMC-R, which is close to the first-best level in each round, similar to behavior observed in treatment NMC. ${ }^{27}$ Figure 4 also shows the misrepresented medium return states and the non-misrepresented medium return states. In the first round, no subject misrepresented any state. Over time, however, the number of faked high return states increases, although it remains well below the maximum number possible. The slow evolution of misrepresentation behavior may result from our cautious way of describing the opportunities for misrepresentation in the instructions to avoid experimenter-demand effects. In addition, subjects may require time to discover the advantages of such misrepresentation, where such misrepresentation is unaccompanied by any disadvantages such as punishment for misreported states, etc. Nevertheless, the finding of much less misrepresentation than is possible is consistent with Mazar et al. (2008), who also observe a rather low incidence of cheating behavior. ${ }^{28}$

\footnotetext{
${ }^{27}$ Average effort in treatment NMC-R does not significantly differ from the theoretical prediction in any of the fifteen rounds; two-tailed $t$-tests, $p>0.257$; two-tailed sign-test, $p>0.307$.

${ }^{28}$ Alternatively, our finding of little misrepresentation may follow from an experimenter demand effect, introduced by our instruction wording "The capital provider can distinguish between a low revenue and other revenues, but not between an intermediate revenue and a high revenue. Therefore if the project revenue is low, this is reported to the capital provider automatically. But if the project revenue is not low, then you have to report to the capital provider if the project attained an intermediate revenue or a high revenue.", as suggested by a reviewer. If this is the case, then we would underestimate the disadvantages, which reinforces our conclusions from treatment NMC-R, as summarized in Result 6.
} 


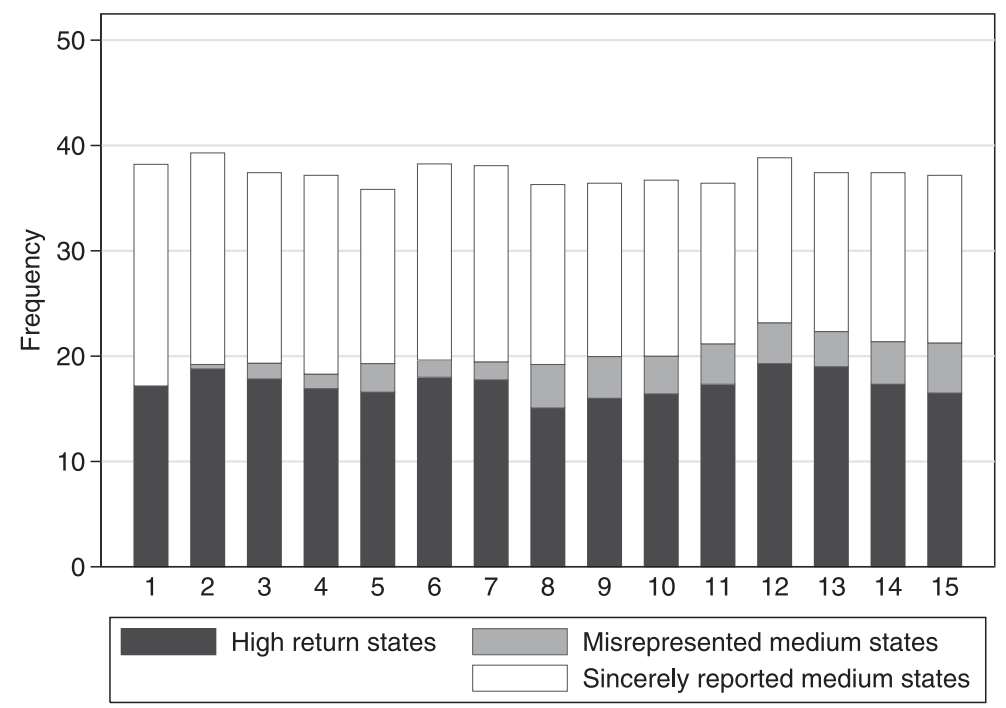

The figure depicts the number of realized high return states averaged over subjects by round. It also indicates the number of misrepresented medium return states as grey-colored bars that are stacked on the bars that represent high returns. Similarly, the outlined bars show the number of non-misrepresented medium return states. The difference between the total number of projects in a round, 50, and the sum of high return states, misrepresented medium return states, and non-misrepresented medium return states indicates the number of low return states as a (non-indicated) residual.

Fig. 4 Average number of realized high return, misrepresented medium, and non-misrepresented medium return states by round

Although the number of misrepresented medium return states is lower than predicted, its implications for payoff redistribution between contracting parties are significant, as shown in Fig. 5. The figure depicts the time paths of average payoffs to entrepreneurs and investors, along with total surplus. Apparently, the increasing number of faked high return states is accompanied by large increases in payoffs to entrepreneurs at the expense of investors. Toward the end of the experiment, entrepreneurs manage to reap amounts from investors that exhaust total surplus. In rounds 11 and 13, (computerized) investor payoffs decline and even become slightly negative. ${ }^{29}$ Most likely, negative or zero payoffs in the contract situation would lead investors to reject the contract. Thus, our results confirm the practical disadvantages of the non-monotonic contract structure, if the environment offers opportunities for state misrepresentation. We summarize our findings as follows:

Result 6 Opportunities for misrepresenting realized return states are partially exploited and erode the participation constraint of the investor through payoff redistribution from investors to entrepreneurs (rejection of the no-misrepresentation Hypothesis 5).

\footnotetext{
${ }^{29}$ This is facilitated by average repayments falling short of the borrowed amount, so that investors partially subsidize project execution.
} 


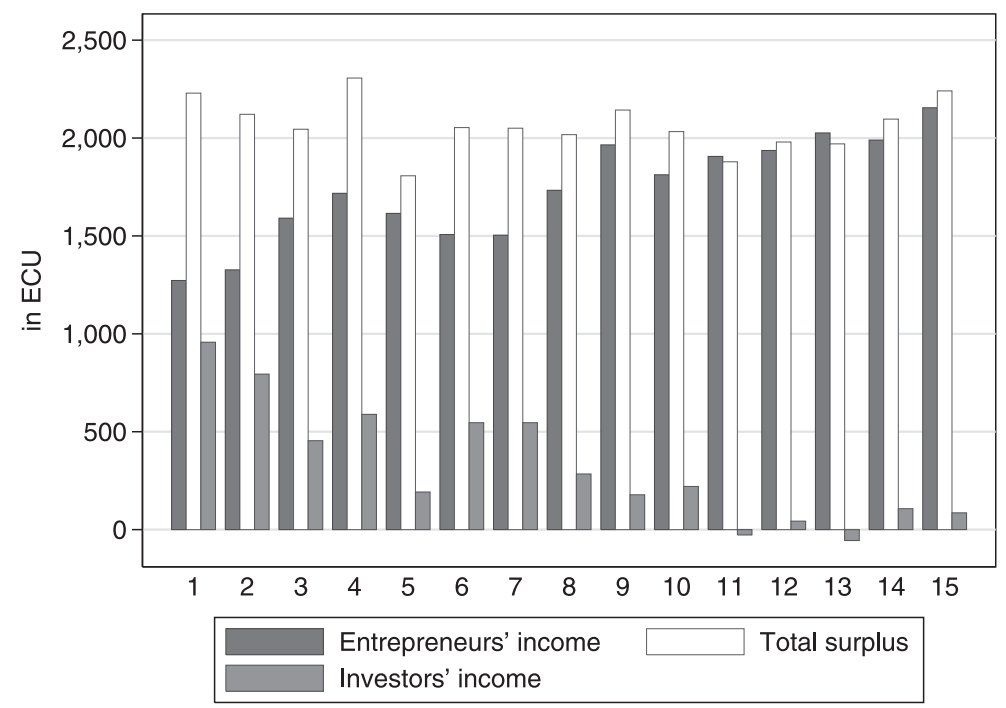

Fig. 5 Average payoffs and total surplus by round in treatment NMC-R

\subsection{Monotonic-contracts hypothesis}

Innes (1990) has shown that the standard debt contract maximizes total surplus in the class of monotonic contracts. In particular, a standard debt contract fares better, theoretically, than a repayment-equivalent equity contract. In the following we compare behavior observed in treatments SDC2 and EQUI2. Both contracts yield the same expected repayment to the investor but differ in their predictions of effort by roughly ten units. While theory predicts 21.3 units of effort under contract SDC2, it predicts 11.4 units under contract EQUI2. The implied difference in total surplus is approximately 403 ECU (see Table 2 for the theoretical predictions under each contract).

Figure 6 depicts the time paths of average effort in either treatment. As seen in the figure, average effort under SDC2 and EQUI2 is similar to that under our basic treatments, SDC and EQUI: In both extension treatments, average effort is at a similar level in the first round (two-tailed $t$-test, $p=0.599)^{30}$ and exceeds the predicted effort (two-tailed $t$-tests, for either treatment $p<0.001$ ). ${ }^{31}$ Over the course of the experiment, as subjects gain experience with contract incentives, average effort under either contract declines and approaches the theoretically predicted level. In either treatment, convergence to the theoretical prediction appears to be partial, as average effort remains somewhat above the theoretical level; in either treatment and in each round, the two-tailed $t$-test shows significant differences, with $p<0.033$. However, average effort appears to be partly affected by a few subjects who continue to occasionally try out larger effort levels at the end of the experiment. Controlling for this aspect by examining median behavior reveals that the medians of effort choices

\footnotetext{
${ }^{30}$ Two-tailed MWU, $p=0.482$.

${ }^{31}$ Two-tailed sign tests, $p<0.007$.
} 


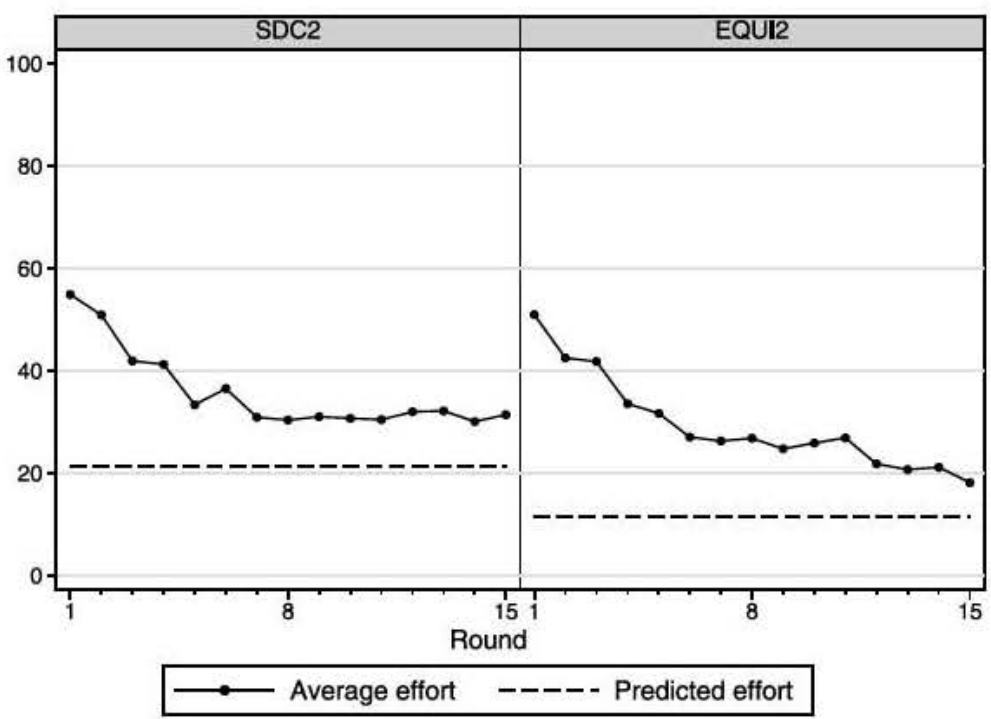

The figure depicts average effort (solid lines) over the rounds of the experiment under the standard debt contract SDC2 (left) and under outside equity EQUI2 (right). The theoretical effort prediction is indicated by dashed lines.

Fig. 6 Average effort and predicted effort by round

converge to the theoretical prediction by round 5 in treatment SDC2 (two-tailed sign test, for rounds $1-4 p<0.064$ and for rounds $5-15, p>0.151$ ) and by round 14 in treatment EQUI2 (two-tailed sign test, for rounds $1-13 p<0.023$; and for rounds $14-15, p>0.151)$.

To test the monotonic-contracts hypothesis, we examine the difference between average effort across both treatments. The graphs of average effort suggest that they are rather similar for rounds $1-11$ and different for rounds $12-15$. Overall, a treatment comparison of average effort (where, for each individual, the average is taken over all rounds) shows insignificant differences (two-tailed $t$-test, $p=0.106$ ). ${ }^{32}$ Roundwise comparisons show that the treatment differences of average effort are insignificant for rounds 1-11 (two-tailed $t$-test, $p>0.105$ ) ${ }^{33}$ For each of the final four rounds, $12-15$, there are significant differences (two-tailed $t$-test, $p<0.065$ ). ${ }^{34}$ Indeed, the finding of a significant treatment difference at the end of the experiment is confirmed by a regression that better accounts for intra-subject correlations, see Electronic Supplementary Material. We carefully interpret these results, at the end of our experiment, as favoring the monotonic-contracts hypothesis.

Figure 7 shows the total surplus premium of the SDC2 contract over the EQUI2 contract in the second half of the experiment, where the median effort level has roughly converged to the predicted effort level. In line with the monotonic-contracts

\footnotetext{
${ }^{32}$ Two-tailed MWU-test, $p=0.274$.

${ }^{33}$ Two-tailed MWU-test, $p>0.148$.

${ }^{34}$ Two-tailed MWU-test, $p<0.039$, for each round except round 14 , where $p=0.124$.
} 


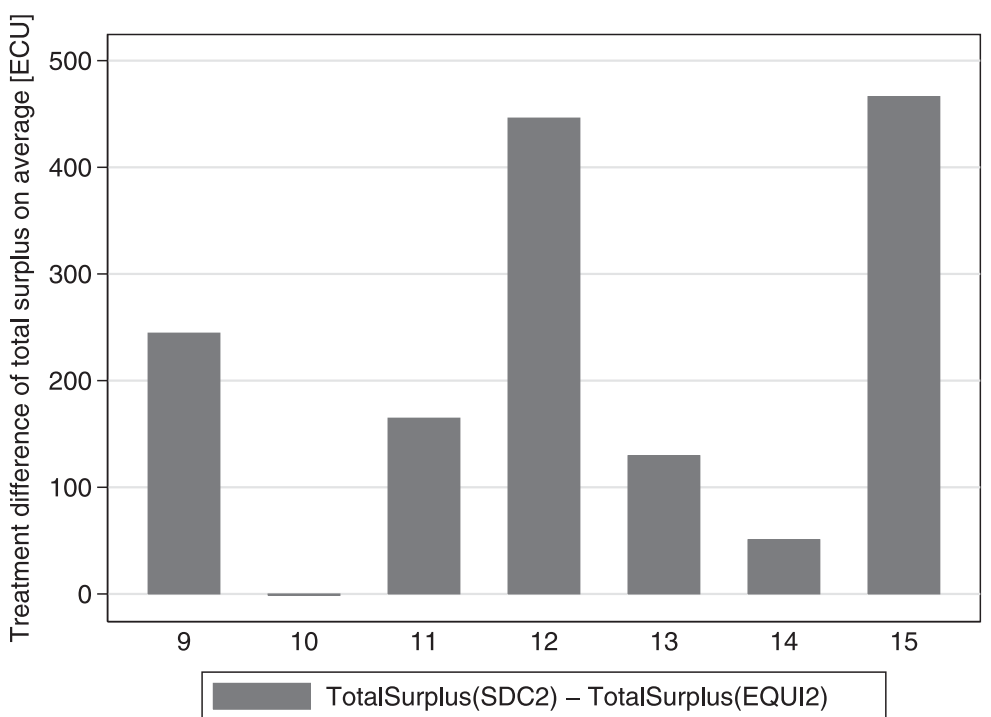

The figure depicts the average difference in total surplus in treatments SDC2 and EQUI2 by round, for rounds $9-15$.

Fig. 7 Total surplus premium of SDC2 over EQUI2 by round

hypothesis, allocative efficiency is higher under the SDC2 contract than under the EQUI2 contract, particularly close to the end of the experiment.

Result 7 With sufficient experience, effort under the standard debt contract SDC2 tends to be larger than that under the repayment-equivalent equity contract EQUI2, implying that allocative efficiency is higher under SDC2 than under EQUI2 (partial support for Hypothesis 4).

\section{Concluding remarks}

We have experimentally examined the incentive effects inherent in funding contracts. Surprisingly, in the early rounds of the experiment, we found no incentive effects at all: the effort level is roughly the same regardless of the contract condition. This finding is robust to raising the stakes accruing to the underlying effort decision as demonstrated by the behavior observed in the one-shot treatment. This result shows that people's abilities to grasp incentive effects through mere introspection are limited. However, as experience with contract conditions accumulates, incentive effects increasingly govern behavior. With sufficient experience, behavior is largely consistent with theoretical predictions, so that the differential incentive effects of funding contracts apply in the long run. As a consequence, we also find support for the non-monotonic-contracts and the monotonic-contracts hypotheses. 
The finding that experience crucially determines how the incentives of funding contracts affect behavior is of particular importance as real-life entrepreneurs, endowed with all sorts of "projects", differ in their experience. For example, an entrepreneur requiring external finance to start a project is inexperienced in the implications of funding contracts at the start of the entrepreneurial career. Our results suggest that no efficiency loss arises with standard debt or equity in these cases, due to limited introspection. The inexperienced entrepreneurs, however, suffer from their inexperience, as they receive lower incomes than predicted. ${ }^{35}$ Depending on the individual entrepreneur and the particular project(s) encountered, an entrepreneur may accumulate experience with the funding contract incentives over the course of a career. We provide evidence that under the standard debt and equity contracts, inefficiencies arise with experienced rather than inexperienced entrepreneurs. Replacing such contracts with non-monotonic contracts would mitigate the losses in allocative efficiency associated with them. One possibility would be a non-monotonic contract that combines a standard debt contract with bonus payments from the investor to the entrepreneur, conditional on achieving relatively high return states.

Importantly, our positive assessment of the non-monotonic contract crucially relies on costless state verification and its enforcement. If state verification is difficult, then the contracted repayment from non-monotonic contracts can be easily manipulated through false state reporting. In our experiment, we observed misrepresentation of states along these lines. Although the amount of misrepresentation was surprisingly small compared to the maximum amount possible-which would be rational for self-interested individuals - it was sufficient to erode the participation constraint of investors through substantially decreased repayments. Nevertheless, state misrepresentation had no negative effects on allocative efficiency, as effort levels remained close to the first-best level and state misrepresentation implied income redistribution from investors to entrepreneurs only.

If non-monotonic contracts are not feasible, then any repayment contract is restricted to specifying (weakly) higher repayments with higher project returns, as with outside equity and standard debt contracts. Theoretically, outside equity is less efficient than a repayment-equivalent standard debt contract; we observed this result in our experiment. Once entrepreneurial subjects acquired sufficient experience with contract incentives, they exert less entrepreneurial effort when external financing takes the form of outside equity than when it takes the form of loans.

\section{References}

Aghion, P., \& Howitt, P. (1992). A model of growth through creative destruction. Econometrica, 60(2), 323-351.

Aghion, P., \& Howitt, P. (1998). Endogenous growth theory. Cambridge: MIT Press.

\footnotetext{
${ }^{35}$ Of course, in real life, inexperienced entrepreneurs can also access other sources of information, which can mitigate the losses due to minimal experience with contract incentives. Cases in point are start-up consultancies and financial advisories.
} 
Baker, M., \& Wurgler, J. (2012). Behavioral corporate finance: an updated survey. In G. M. Constantinides, M. Harris, \& R. M. Stulz (Eds.), Handbook of the economics of finance (Vol. 2). Amsterdam: NorthHolland.

Baker, M., Ruback, R. S., \& Wurgler, J. (2007). Behavioral corporate finance: a survey. In B. Espen Eckbo (Ed.), Handbook in corporate finance: empirical corporate finance. Amsterdam: North-Holland.

Barro, R. J., \& Sala-i-Martin, X. (2003). Economic growth (2nd ed.). Cambridge: MIT Press.

Brandt, J., \& Charness, G. (2004). Do labour market conditions affect gift exchange? Some experimental evidence. The Economic Journal, 114, 684-708.

Brown, M., \& Zehnder, C. (2007). Credit registries, relationship banking and loan repayment. Journal of Money, Credit, and Banking, 39(8), 1883-1918.

Brown, M., \& Zehnder, C. (2010). The emergence of information sharing in credit markets. Journal of Financial Intermediation, 19(2), 255-278.

DeJong, D. V., Forsythe, R., \& Lundholm, R. J. (1985). Ripoffs, lemons, and reputation formation in agency relationships: a laboratory market study. The Journal of Finance, 40(3), 809-820.

Fehr, E., \& Zehnder, C. (2006). Reputation and credit market formation (NCCR Finrisk Working Paper No. 179).

Fehr, E., Kirchsteiger, G., \& Riedl, A. (1993). Does fairness prevent market clearing? An experimental investigation. The Quarterly Journal of Economics, 108(2), 437-460.

Fehr, E., Klein, A., \& Schmidt, K. M. (2007). Fairness and contract design. Econometrica, 75(1), 121154.

Fischbacher, U. (2007). z-Tree: Zurich toolbox for ready-made economic experiments. Experimental Economics, 10, 171-178.

Fischbacher, U., \& Föllmi-Heusi, F. (2013). Lies in disguise. Journal of the European Economic Association, 11, 525-547.

Greiner, B. (2004). The Online Recruitment System ORSEE 2.0-a guide for the organization of experiments in economics (Working Paper Series in Economics, No. 10). University of Cologne.

Innes, R. T. (1990). Limited liability and incentive contracting with ex ante action choices. Journal of Economic Theory, 52, 45-67.

Irlenbusch, B., \& Sliwka, D. (2005). Transparency and reciprocal behavior in employment relations. Journal of Economic Behavior \& Organization, 56(3), 383-403.

Kirchkamp, O., Reiß, J. P., \& Sadrieh, K. (2008). A pure variation of risk in first-price auctions (METEOR Research Memorandum 08/050).

Lazear, E. (2000). Performance pay and productivity. The American Economic Review, 90(5), 1346-1361.

Malmendier, U., \& Tate, G. (2005). CEO overconfidence and corporate investment. The Journal of Finance, $L X(6), 2661-2700$

Mazar, N., Amir, O., \& Ariely, D. (2008). The dishonesty of honest people: a theory of self-concept maintenance. Journal of Marketing Research, XLV, 633-644.

Milgrom, P. R. (1981). Good news and bad news: representation theorems and applications. Bell Journal of Economics, 13, 369-378.

Niemeyer, C., Reiß, J. P., \& Sadrieh, K. (2013). Reducing risk in experimental games and individual choice (Working Paper). Karlsruhe Institute of Technology.

Reiß, J. P., \& Wolff, I. (2012). Incentive effects of funding contracts: an experiment (TWI Research Paper Series, No. 78). University of Konstanz.

Reiß, J. P., \& Wolff, I. (2013). Funding of entrepreneurs: an experimental approach (Working Paper, Mimeo).

Rogers, W. H. (1993). Regression standard errors in clustered samples. Stata Technical Bulletin, 13, 19-23. Reprinted in Stata Technical Bulletin Reprints, 3, 88-94.

Romer, P. M. (1990). Endogenous technological change. Journal of Political Economy, 98, S71-S102.

Serra-Garcia, M. (2010). Moral hazard in credit markets: the incentive effect of collateral (Working Paper). Tilburg University.

Shearer, B. (2004). Piece rates, fixed wages and incentives: evidence from a field experiment. Review of Economic Studies, 71(2), 513-534. 